\title{
Reabilitação cinesioterapêutica em um homem com artrite reumatoide
}

\author{
Kinesiotherapeutic rehabilitation in a man with rheumatoid arthritis
}

BRUNA KNOB ${ }^{\mathrm{I}}$

MATHEUS SANTOS GoMes JORGE

FERNANDA LORENZET BRESOLIN ${ }^{1}$

Leticia AParecida Bolzan ${ }^{I}$

DAFNe dos SANTOS RiBEIRo ${ }^{1}$

Caroline Zanin

Lia Mara Wibelinger ${ }^{1}$

${ }^{\mathrm{I}}$ Universidade de Passo Fundo

(UPF), Passo Fundo/RS - Brasil.
Resumo A artrite reumatoide é uma doença reumática, que acomete preferencialmente mulheres e pode causar agravos musculoesqueléticos como dores articulares, diminuição do trofismo muscular e impacto na qualidade de vida dos indivíduos. O objetivo deste estudo foi verificar os efeitos da reabilitação cinesioterapêutica em um homem com artrite reumatoide. Trata-se de um relato de caso de um homem com artrite reumatoide, com 49 anos de idade, cuja queixa principal eram dores articulares diversas. Ele realizou 26 sessões de fisioterapia, ancoradas em cinesioterapia, com exercícios de mobilizações articulares, alongamentos musculares, fortalecimentos musculares, exercícios de transferência de peso, de equilíbrio e de marcha, terapia manual e crioterapia, onde utilizou vários recursos. Após a intervenção fisioterapêutica, observou-se diminuição dos pontos dolorosos e da intensidade da dor, aumento do trofismo muscular dos braços e da coxa direita e melhora de cinco dos oito domínios da qualidade de vida do indivíduo estudado (limitação por aspectos emocionais, dor, saúde mental, capacidade funcional e a vitalidade, respectivamente). Concluiu-se que o plano cinesioterapêutico proposto foi benéfico para a diminuição da dor, aumento do trofismo muscular e melhora da qualidade de vida de um homem com artrite reumatoide.

Palavras-Chave: Artrite reumatoide. Dor. Sistema musculoesQUelÉtico. Qualidade DE VIDA. Fisioterapia. Exercício.

Aвstract Rheumatoid arthritis is a rheumatic disease, which usually affects women and may cause musculoskeletal disorders such as joint pain, decreased muscle trofism and impact on individual's quality of life. The aim of this study was to verify the effects of kinesiotherapeutic rehabilitation in a man with rheumatoid arthritis. This is a case report of a man with rheumatoid arthritis, 49 years old, whose main complaint was various joint pains. The individual performed 26 physiotherapy sessions, based on kinesiotherapya, with exercises of joint mobilization, muscle stretching, muscle strengthening, weight transfer, balance and gait exercises, manual therapy and cryotherapy, where several resources were used. It was observed tender points and pain intensity decreased, muscle trofism increased in the arms and right thigh, and improvement in five of the eight domains of the individual's quality of life (limitation by emotional aspects, pain, mental health, functional capacity and vitality, respectively). After the physiotherapeutic intervention, it was concluded that the kinesiotherapeutic plan proposed was beneficial for pain reduction, increased muscle trophism and improvement of the quality of life of a man with rheumatoid arthritis.

Key-Words: Rheumatoid arthritis. Pain. Musculoskeletal system. Quality of Life. Physical therapy specialty. Exercise. 


\section{INTRODUÇÃO}

A artrite reumatoide (AR) é uma doença reumática autoimune, de etiologia idiopática e caráter crônico-inflamatório. Acomete os tecidos, órgãos e, principalmente, as articulações periféricas de maneira simétrica, gerando deformidades articulares incapacitantes. ${ }^{1}$ Ocorre em todos os grupos étnicos, e a prevalência é de aproximadamente $1 \%$ da população brasileira e mundial, com predomínio no gênero feminino, entre as idades de 20 e 60 anos. $^{2}$ São pouco comuns os casos de indivíduos do gênero masculino acometidos pela doença. ${ }^{1}$

O quadro clínico da doença é composto por rigidez matinal ou após longos períodos de imobilização (com pelo menos uma hora de duração), fadiga, mal-estar, edema articular simétrico dos tecidos moles, perda de força e resistência muscular, descondicionamento físico, presença de nódulos reumatoides e do fator reumatoide em exame clínico e erosões radiográficas e/ou osteopenia periarticular.,

Acompanhado a isso, a dor é a queixa principal relatada pelos indivíduos acometidos. ${ }^{5}$ Em doenças reumáticas, como a AR, os agravos musculoesqueléticos podem ser fatores que impliquem nas condições musculares. As deformidades articulares podem interferir na geração de torque máximo, assim como, a imobilização pode gerar encurtamento muscular, a miosite pode causar enfraquecimento muscular e a dor e o derrame articular podem gerar inibição reflexa em posição de defesa. Como consequências, ocorrem perda de força muscular e atrofia dos músculos envolvidos. ${ }^{1}$

Os indivíduos com AR podem enfrentar desafios ocupacionais em circunstância dos impactos negativos produzidos pela doença sobre a vida pessoal e profissional, exacerbando o quadro sintomatológico experimentado por estes. Enquanto para as mulheres com AR os conflitos pessoais e a sobrecarga são fatores comuns no ambiente de trabalho, para os homens os principais problemas enfrentados são a insatisfação para com as suas tarefas, a redução do seu desempenho durante as suas atividades laborativas, o aumento do absentismo ao trabalho e dos casos de depressão. ${ }^{6}$

Quando a doença é diagnosticada, o tratamento objetiva evitar as lesões articulares, minimizar a agressão e sua atividade, evitar a progressão dos danos e os agravos osteomioarticulares já estabelecidos. Apesar disso, a condição de exacerbação e remissão da AR é um fator que pode interferir no tratamento do indivíduo portador., ${ }^{7,8}$

A reabilitação efetiva deve ser pautada na sua realização adequada, impedindo, assim, que danos articulares tornem-se irreversíveis. A fisioterapia atua em todas as fases da doença, diminuindo a dor, mantendo ou recuperando a mobilidade articular e prevenindo as atrofias musculares e as deformidades articulares. ${ }^{1}$ Para isso, as modalidades de exercício físico terapêutico, por meio da cinesioterapia, apresentam bons resultados sobre a força muscular, a funcionalidade, a capacidade cardiorrespiratória, a disposição e o retorno às atividades funcionais dos indivíduos com AR. Embora existam evidências de melhora na condição geral, ainda as técnicas ideais e os seus resultados não são claros de forma específica. ${ }^{9}$

Assim, o objetivo do presente estudo foi verificar os efeitos da reabilitação cinesioterapêutica em um homem com AR.

\section{Relato DE CASO}

Trata-se de um relato de caso, que faz parte de um projeto denominado "Efeitos do tratamento fisioterapêutico em pacientes portadores de doenças reumáticas", aprovado pelo Comitê de Ética em Pesquisa em Seres 
Humanos da Universidade de Passo Fundo, sob protocolo $\mathrm{n}^{\circ}$. 348.381, conforme determina a Declaração de Helsinki de 1975, revisada em 2000, e a Resolução do Conselho Nacional de Saúde do Brasil 466/2012.

Selecionou-se para o estudo, por conveniência, um indivíduo do gênero masculino com 49 anos de idade, portador de AR, que estava em uma lista de espera do Setor de Fisioterapia Reumatológica para o tratamento na Clínica de Fisioterapia, localizada nas dependências da Faculdade de Educação Física e Fisioterapia, da Universidade de Passo Fundo, Passo Fundo/RS.

Inicialmente, o indivíduo foi submetido a uma avaliação inicial, que incluiu a coleta de dados pessoais, por meio de um questionário estruturado pelos próprios pesquisadores, a avaliação do exame físico, onde verificou-se a pressão arterial, a frequência cardíaca, a frequência respiratória, a massa corporal e a estatura do participante. $\mathrm{O}$ indivíduo apresentava pressão arterial de 130/90 $\mathrm{mmHg}$, frequência cardíaca de 70 batimentos por minuto, frequência respiratória de 16 ciclos por minuto, massa corporal de $77 \mathrm{~kg}$ e estatura de $1,69 \mathrm{~m}$. As variáveis de desfecho analisadas neste estudo foram a intensidade da dor, o número de pontos dolorosos, o trofismo muscular e a qualidade de vida, por meio de testes e questionários específicos.

A intensidade da dor foi analisada por meio da escala visual analógica, que é linha reta horizontal enumerada de 0 (sem dor) a 10 (pior dor imaginável), onde o indivíduo deve indicar quantitativamente a intensidade da dor presente no momento da avaliação, podendo esta ainda ser quantificada em uma dor leve, moderada ou intensa. ${ }^{10}$ Já o número de pontos dolorosos foi analisado por meio do mapa de dor corporal, uma representação do corpo humano, onde o examinado indica a localização e distribuição específica da dor no momento da avaliação. ${ }^{11}$

A avaliação do trofismo muscular deu-se por meio da perimetria dos braços e das coxas, com uma fita métrica de $150 \mathrm{~cm}$. Por fim, a qualidade de vida foi mensurada por intermédio do Questionário de Qualidade de Vida SF-36, um questionário de 36 itens que avalia os aspectos físicos e mentais do indivíduo analisado. Os escores finais de cada um dos oito domínios do SF-36 vão de 0 (o que corresponde à pior nulidade da qualidade de vida) a 100 (que corresponde à melhor nulidade da qualidade de vida). ${ }^{12}$

A intervenção terapêutica em indivíduos com AR preocupa-se em produzir efeitos a curto prazo sobre o quadro doloroso, a capacidade aeróbica, a percepção de saúde, a funcionalidade e a qualidade de vida, sendo que entre as diversas ferramentas terapêuticas, o fortalecimento muscular e a resistência cardiorrespiratória são os mais indicados, de acordo com a literatura. ${ }^{1,13,14}$ Apesar da heterogeneidade entre os estudos, não há evidência de efeitos adversos produzidos pelo exercício físico, assim, a sua aplicabilidade pode ser considerada segura, promovendo a melhor qualidade da saúde dos indivíduos, especialmente no combate à dor e sem exacerbar o quadro patológico. ${ }^{9}$

O programa de intervenção fisioterapêutica baseou-se na utilização do exercício físico terapêutico, denominado de cinesioterapia. O participante realizou 26 sessões, com frequência de duas sessões semanais, durante 17 semanas, entre os meses de agosto e novembro de 2015.

Com base em estudos já referenciados na literatura, ${ }^{15,16}$ o plano cinesioterapêutico foi delineado visando surtir efeitos sobre a dor, o trofismo muscular e a qualidade de vida do indivíduo do estudo atual. A Tabela 1 apresenta o cronograma do programa de intervenção fisioterapêutica realizado. 
Tabela 1: Cronograma do programa de intervenção fisioterapêutica

\begin{tabular}{|c|c|c|c|}
\hline Sessões & Exercício & Descrição do exercício & Número de séries e repetições \\
\hline $1^{\mathrm{a}}$ à $26^{\mathrm{a}}$ & $\begin{array}{l}\text { Mobilizações } \\
\text { articulares }\end{array}$ & $\begin{array}{l}\text { Realizaram-se mobilizações articulares dos ombros e dos } \\
\text { quadris em solo e dos punhos e dos dedos das mãos em } \\
\text { um turbilhão com água aquecida a } 36^{\circ} \mathrm{C} \text {. }\end{array}$ & - \\
\hline $1^{\mathrm{a}}$ à $26^{\mathrm{a}}$ & $\begin{array}{l}\text { Alongamentos } \\
\text { musculares }\end{array}$ & $\begin{array}{l}\text { Realizaram-se alongamentos lentos e mantidos, de modo } \\
\text { ativo-assistido, dos principais grupos musculares dos } \\
\text { membros superiores e dos membros inferiores, durante } \\
30 \text { segundos cada grupo muscular. }\end{array}$ & - \\
\hline $1^{\mathrm{a}}$ à $26^{\mathrm{a}}$ & $\begin{array}{l}\text { Dissociação } \\
\text { de cinturas } \\
\text { escapular e } \\
\text { pélvica }\end{array}$ & $\begin{array}{l}\text { O indivíduo deveria sentar sobre uma bola suíça de } 65 \\
\mathrm{~cm} \text { e realizar movimento de dissociação das cinturas } \\
\text { escapular e pélvica no sentido anteroposterior e lateral. }\end{array}$ & $\begin{array}{l}\text { Duas séries de } 15 \text { repetições } \\
\left(1^{\mathrm{a}}-10^{\mathrm{a}}\right) \\
\text { Três séries de } 10 \text { repetições } \\
\left(11^{\mathrm{a}}-15^{\mathrm{a}}\right) \\
\text { Quatro séries de oito } \\
\text { repetições }\left(16^{\mathrm{a}}-26^{\mathrm{a}}\right)\end{array}$ \\
\hline $1^{\mathrm{a}}$ à $26^{\mathrm{a}}$ & $\begin{array}{l}\text { Exercícios } \\
\text { resistidos para } \\
\text { as mãos }\end{array}$ & $\begin{array}{l}\text { Realizaram-se exercícios resistidos utilizando-se um } \\
\text { fortalecedor de punhos e dedos em formato de rede } \\
\left(1^{\mathrm{a}} \text { à } 26^{\mathrm{a}}\right) \text {, massas de modelar terapêuticas }\left(1^{\mathrm{a}} \text { à } 26^{\mathrm{a}}\right) \\
\text { e exercícios de oponência dos dedos }\left(1^{\mathrm{a}} \text { à } 26^{\mathrm{a}}\right) \text { para } \\
\text { fortalecer as mãos e os dedos do indivíduo. }\end{array}$ & $\begin{array}{l}\text { Duas séries de } 15 \text { repetições } \\
\left(1^{\mathrm{a}}-8^{\mathrm{a}}\right) \\
\text { Três séries de } 10 \text { repetições } \\
\left(9^{\mathrm{a}}-15^{\mathrm{a}}\right) \\
\text { Quatro séries de oito } \\
\text { repetições }\left(16^{\mathrm{a}}-26^{\mathrm{a}}\right)\end{array}$ \\
\hline $1^{\mathrm{a}}$ à $8^{\mathrm{a}}$ & $\begin{array}{l}\text { Exercícios } \\
\text { respiratórios }\end{array}$ & $\begin{array}{l}\text { Realizaram-se exercícios respiratórios em padrão } \\
\text { diafragmático profundo associado à elevação dos } \\
\text { membros superiores com um bastão de madeira sem } \\
\text { carga. }\end{array}$ & $\begin{array}{l}\text { Duas séries de } 10 \text { repetições } \\
\left(1^{\mathrm{a}}-3^{\mathrm{a}}\right) \\
\text { Três séries de } 10 \text { repetições } \\
\left(4^{\left.\mathrm{a}-8^{\mathrm{a}}\right)}\right.\end{array}$ \\
\hline $9^{\mathrm{a}}$ à $13^{\mathrm{a}}$ & $\begin{array}{l}\text { Exercício de } \\
\text { ponte }\end{array}$ & $\begin{array}{l}\text { Realizou-se exercício de ponte, do método Bobath sobre } \\
\text { um tatame. }\end{array}$ & $\begin{array}{l}\text { Três séries de } 10 \text { repetições } \\
\left(9^{a}-13^{a}\right)\end{array}$ \\
\hline $\begin{array}{l}14^{\mathrm{a}} \text { à } \\
19^{\mathrm{a}}\end{array}$ & $\begin{array}{l}\text { Treino de } \\
\text { Equilíbrio }\end{array}$ & $\begin{array}{l}\text { Treino de equilíbrio em dispositivos proprioceptivos, } \\
\text { como balance pad de espuma }\left(14^{\mathrm{a}} \text { à } 16^{\mathrm{a}}\right) \text {, balance pad } \\
\text { emborrachado em formato de disco }\left(15^{\mathrm{a}} \text { à } 17^{\mathrm{a}}\right) \text {, balancim } \\
\left(16^{\mathrm{a}} \text { à } 18^{\mathrm{a}}\right) \text {, trampolim }\left(17^{\mathrm{a}} \text { à } 19^{\mathrm{a}}\right) \text { e pranchas de Freeman } \\
\left(18^{\mathrm{a}} \text { e } 19^{\mathrm{a}}\right) \text {. }\end{array}$ & - \\
\hline $\begin{array}{l}20^{\mathrm{a}} \text { à } \\
26^{\mathrm{a}}\end{array}$ & $\begin{array}{l}\text { Treino de } \\
\text { marcha }\end{array}$ & $\begin{array}{l}\text { Treino de caminhada em diferentes tipos de solos, em } \\
\text { rampas e em escadas; }\end{array}$ & - \\
\hline $1^{\mathrm{a}}$ à $26^{\mathrm{a}}$ & $\begin{array}{l}\text { Exercícios } \\
\text { resistidos para } \\
\text { os membros } \\
\text { superiores }\end{array}$ & $\begin{array}{l}\text { Realizaram-se exercícios resistidos utilizando-se faixas } \\
\text { elásticas }\left(1^{\mathrm{a}} \text { à } 13^{\mathrm{a}}\right) \text { nas cores rosa }\left(1^{\mathrm{a}} \text { à } 3^{\mathrm{a}}\right) \text {, verde }\left(4^{\mathrm{a}} \text { à }\right. \\
\left.6^{\mathrm{a}}\right) \text {, azul }\left(7^{\mathrm{a}} \text { à } 9^{\mathrm{a}}\right) \text { e roxa }\left(10^{\mathrm{a}} \text { à } 13^{\mathrm{a}}\right) \text { e halteres }\left(14^{\mathrm{a}} \text { à } 26^{\mathrm{a}}\right) \\
\text { de } 0,5 \mathrm{~kg}\left(14^{\mathrm{a}} \text { à } 17^{\mathrm{a}}\right), 1 \mathrm{~kg}\left(18^{\mathrm{a}} \text { à } 20^{\mathrm{a}}\right), 1,5 \mathrm{~kg}\left(21^{\mathrm{a}} \text { à } 23^{\mathrm{a}}\right) \\
\text { e } 2 \mathrm{~kg}\left(24^{\mathrm{a}} \text { à } 26^{\mathrm{a}}\right) \text { para fortalecer os músculos flexores, } \\
\text { extensores, abdutores, adutores, rotadores internos e } \\
\text { rotadores externos dos ombros e flexores e extensores } \\
\text { dos cotovelos. }\end{array}$ & $\begin{array}{l}\text { Três séries de } 10 \text { repetições } \\
\left(1^{\mathrm{a}} \text { à } 26^{\mathrm{a}}\right)\end{array}$ \\
\hline $1^{\mathrm{a}}$ à $26^{\mathrm{a}}$ & $\begin{array}{l}\text { Exercícios } \\
\text { resistidos para } \\
\text { os membros } \\
\text { inferiores }\end{array}$ & $\begin{array}{l}\text { Realizaram-se exercícios resistidos utilizando-se faixas } \\
\text { elásticas }\left(1^{\mathrm{a}} \text { à } 13^{\mathrm{a}}\right) \text { nas cores rosa }\left(1^{\mathrm{a}} \text { à } 3^{\mathrm{a}}\right) \text {, verde }\left(4^{\mathrm{a}} \text { à } 6^{\mathrm{a}}\right) \text {, } \\
\text { azul }\left(7^{\mathrm{a}} \text { à } 9^{\mathrm{a}}\right) \text { e roxa }\left(10^{\mathrm{a}} \text { à } 13^{\mathrm{a}}\right) \text { e caneleiras }\left(14^{\mathrm{a}} \text { à } 26^{\mathrm{a}}\right) \text { de } \\
1 \mathrm{~kg}\left(14^{\mathrm{a}} \text { à } 17^{\mathrm{a}}\right), 1,5 \mathrm{~kg}\left(18^{\mathrm{a}} \text { à } 21^{\mathrm{a}}\right) \text { e } 2 \mathrm{~kg}\left(22^{\mathrm{a}} \text { à } 26^{\mathrm{a}}\right) \text { para } \\
\text { fortalecer os músculos flexores, extensores, abdutores e } \\
\text { adutores dos quadris e flexores e extensores dos joelhos. }\end{array}$ & $\begin{array}{l}\text { Três séries de } 10 \text { repetições } \\
\left(1^{\mathrm{a}} \text { à } 26^{\mathrm{a}}\right)\end{array}$ \\
\hline $1^{\mathrm{a}}$ à $26^{\mathrm{a}}$ & $\begin{array}{l}\text { Terapia } \\
\text { manual }\end{array}$ & $\begin{array}{l}\text { Realizaram-se pompagens na região cervical do } \\
\text { indivíduo nos } 5 \text { minutos finais das sessões. }\end{array}$ & - \\
\hline- & Crioterapia & $\begin{array}{l}\text { Realizou-se crioterapia durante } 20 \text { minutos em locais } \\
\text { onde o indivíduo relatava dor ao final da sessão. }\end{array}$ & - \\
\hline
\end{tabular}


Ao final das sessões, os parâmetros dor, trofismo muscular e qualidade de vida foram reavaliados e os dados registrados no programa Microsoft Excel 2010.

\section{RESUltados}

$\mathrm{Na}$ entrevista pré-seletiva, identificou-se que o indivíduo tinha 49 anos de idade, era casado e tinha dois filhos. Possuía diagnóstico clínico de AR havia 13 anos, apresentava histórico de doença reumática na família e hiperuricemia como doença associada. Fazia uso de cinco medicamentos contínuos e manteve esse tratamento durante a intervenção fisioterapêutica. Com relação às condições físicas do indivíduo, este relatava como queixa principal as dores articulares em diversos pontos do corpo, principalmente nas mãos. Sua marcha era independente e sem padrões patológicos.
Apresentava crepitações no ombro direito e nos joelhos, redução da força muscular generalizada e da amplitude de movimento articular, devido à deformidade e às dores produzidas pela patologia.

A Tabela 2 apresenta os resultados referentes ao número de pontos dolorosos e à intensidade da dor do indivíduo estudado nas fases pré e pós-intervenção cinesioterapêutica.

Houve diminuição do número de pontos dolorosos, que passaram de 13 , na fase pré-intervenção, para oito, na fase pós-intervenção. Além disso, houve diminuição da intensidade da dor em três pontos, passando de um quadro de dor moderada para um quadro de dor leve.

A Tabela 3 apresenta os resultados referentes ao trofismo muscular dos braços e das coxas do indivíduo estudado nas fases pré e pós-intervenção cinesioterapêutica.

Tabela 2: Dor pré e pós-intervenção cinesioterapêutica

\begin{tabular}{lll} 
& \multicolumn{1}{c}{ Pré-intervenção } & \multicolumn{1}{c}{ Pós-intervenção } \\
\hline Número & - Punhos direito e esquerdo (dois pontos) & - Punhos direito e esquerdo (dois pontos) \\
de pontos & - Ombros direito e esquerdo (dois pontos) & - Ombro direito (um ponto) \\
de dor & - Região cervical (um ponto) & - Quadril esquerdo (um ponto) \\
& - Quadris direito e esquerdo (dois pontos) & - Joelhos direito e esquerdo (dois pontos) \\
& - Joelhos direito e esquerdo (dois pontos) & - Pés direito e esquerdo (dois pontos) \\
& - Tornozelos direito e esquerdo (dois pontos) & \\
Dor** & - Pés direito e esquerdo (dois pontos) & \\
\hline
\end{tabular}

Legenda: ${ }^{*}$ (de acordo com o mapa de dor corporal); ${ }^{* *}$ (de acordo com a escala visual analógica)

Tabela 3: Trofismo muscular pré e pós-intervenção cinesioterapêutica

\begin{tabular}{lcccc} 
& \multicolumn{2}{c}{ Lado direito } & \multicolumn{2}{c}{ Lado esquerdo } \\
\hline \multicolumn{1}{c}{ Local } & Pré-intervenção & Pós-intervenção & Pré-intervenção & Pós-intervenção \\
\hline $\begin{array}{l}\text { Braço }(10 \mathrm{~cm} \text { acima do } \\
\text { olecrano) }\end{array}$ & $28 \mathrm{~cm}$ & $29 \mathrm{~cm}$ & $27 \mathrm{~cm}$ & $31 \mathrm{~cm}$ \\
$\begin{array}{l}\text { Braço }(15 \mathrm{~cm} \text { acima do } \\
\text { olecrano) }\end{array}$ & $29 \mathrm{~cm}$ & $31 \mathrm{~cm}$ & $29 \mathrm{~cm}$ & $31 \mathrm{~cm}$ \\
$\begin{array}{l}\text { Coxa (10 cm acima do } \\
\text { centro da patela) }\end{array}$ & $43 \mathrm{~cm}$ & $46 \mathrm{~cm}$ & $44 \mathrm{~cm}$ & $44 \mathrm{~cm}$ \\
$\begin{array}{l}\text { Coxa (15 cm acima do } \\
\text { centro da patela) }\end{array}$ & $48 \mathrm{~cm}$ & $48 \mathrm{~cm}$ & $49 \mathrm{~cm}$ & $49 \mathrm{~cm}$ \\
\hline
\end{tabular}


Observou-se aumento do trofismo muscular em cinco das oito medições realizadas, sendo elas nos 10 e $15 \mathrm{~cm}$ acima do olecrano em ambos os braços e nos $10 \mathrm{~cm}$ acima do centro da patela da coxa direita.

A Tabela 4 apresenta os resultados referentes à qualidade de vida do indivíduo estudado nas fases pré e pós-intervenção cinesioterapêutica.

\begin{tabular}{lcc}
\hline Domínios & Pré-intervenção & Pós-intervenção \\
\hline Capacidade funcional & 20 pontos & 35 pontos \\
Limitação por aspectos físicos & 0 pontos & 0 pontos \\
Dor & 30 pontos & 51 pontos \\
Estado geral de saúde & 52 pontos & 42 pontos \\
Vitalidade & 35 pontos & 50 pontos \\
Aspectos sociais & 62,5 pontos & 62,5 pontos \\
Limitação por aspectos emocionais & 0 pontos & 33,33 pontos \\
Saúde metal & 56 pontos & 72 pontos \\
\hline
\end{tabular}

Em linhas gerais, houve melhora em cinco dos oito domínios avaliados que constituem a qualidade de vida, sendo eles a limitação por aspectos emocionais, a dor, a saúde mental, a capacidade funcional e a vitalidade, respectivamente.

\section{Discussão}

A AR caracteriza-se pela cronicidade, degeneração articular e processo inflamatório persistente, podendo atingir diversos órgãos, tecidos e articulações. O principal impacto é articular, com envolvimento da membrana sinovial, causando uma sinovite não supurativa e evolução para erosões cartilaginosas e ósseas, comprometendo a funcionalidade e integridade das mesmas. ${ }^{17}$ A maioria dos indivíduos com AR é do gênero feminino, na faixa etária dos 50 aos 69 anos, cuja queixa principal é a dor e faz uso de medicamentos contínuos. Além disso, muitos dos indivíduos possuíam algum tipo de doença associada e histórico familiar de doença reumática. ${ }^{5} \mathrm{O}$ que vai de encontro ao estudo atual, haja vista que o indivíduo estudado era do gênero masculino e estava com 49 anos de idade, sendo que o seu diagnóstico clínico foi realizado havia 13 anos. Por sua vez, alguns aspectos concordam com o presente estudo, pois o indivíduo também apresentava como queixa principal a dor, fazia uso de medicamentos contínuos e possuía doença associada e histórico familiar de doença reumática.

Os indivíduos com AR possuem tendência à diminuição da capacidade aeróbica, da resistência e da força muscular. Desse modo, a fisioterapia torna-se fundamental para eles, tanto na fase aguda, quanto na fase crônica da doença. Particularmente, a abordagem cinesioterapêutica reduz as dores articulares, o processo inflamatório e a rigidez matinal, promovendo a melhora do estado geral. São indicados exercícios respiratórios, mobilizações articulares, exercícios de fortalecimento (inicialmente de modo isométrico, progredindo para exercícios de modos concêntrico e excêntrico) e exercícios de amplitude de movimento para ativar o fluxo sanguíneo e melhorar a nutrição das articulações imobilizadas. ${ }^{1}$ Com base nisto, o programa adotado como intervenção neste estudo foi a cinesioterapia, realizada no in- 
tuito de mobilizar as articulações, diminuir o quadro doloroso, fortalecer a musculatura e, por conseguinte, permitir ao indivíduo realizar suas tarefas, ofertando-lhe melhor qualidade de vida, onde constataram-se efeitos benéficos sobre tais aspectos.

Fisiologicamente, a realização de exercícios físicos pode promover uma elevação do limiar de dor, por meio da liberação de opioides endógenos e da inibição do mecanismo nociceptivo cerebral. Assim, mesmo pela ativação de musculatura não acometida dolorosamente, há efeito sistêmico de analgesia no indivíduo com $\mathrm{AR},{ }^{18}$ o que concorda com os achados do presente estudo, pois a intervenção cinesioterapêutica adotada neste estudo foi capaz de produzir efeitos benéficos sobre o quadro doloroso relatado pelo indivíduo. Também foi possível constatar a diminuição da intensidade da dor e do número de pontos dolorosos na fase pós-intervenção.

O indivíduo estudado apresentou queixa de dores articulares em pontos diversos, especialmente nas mãos, o que concorda com a literatura, pois a AR pode produzir dor e perda da função das mãos, podendo levar a limitações das atividades, restrições de participação do indivíduo nas atividades sociais e redução da qualidade de vida. ${ }^{19}$ Dessa forma, pode ocorrer redução na força de preensão palmar bilateral, que tende a regredir em condições inversamente proporcionais à evolução da atividade da doença, impossibilitando os indivíduos com AR de produzirem a força de preensão palmar máxima. ${ }^{20}$ Nesse sentido, o impacto sobre as mãos do indivíduo poderia ser um fator desencadeador da redução da funcionalidade, diminuição da atividade muscu- lar e, consequente, diminuição do trofismo muscular para os membros superiores na fase pré intervenção.

Ainda não há um tratamento ideal para o comprometimento e a disfunção das mãos e dos membros superiores frequentemente observados nos indivíduos com AR, sendo indispensável uma intervenção individualizada e personalizada para cada indivíduo. ${ }^{21}$ Porém, os exercícios passivos, como a mobilização articular, os exercícios de fortalecimento com materiais diversos e as atividades de motricidade fina são recursos utilizados para a reabilitação das mãos dos indivíduos com $\mathrm{AR},{ }^{1}$ o que corrobora com os achados do presente estudo, onde foram utilizados exercícios para as mãos, como as mobilizações articulares e o fortalecimento muscular, e se constatou melhora do trofismo muscular após a intervenção.

Um estudo de caso realizado com um indivíduo com AR submetido a um programa de intervenção fisioterapêutica, ancorada em cinesioterapia, envolveu o uso de exercícios resistidos para as mãos, técnica de Facilitação Neuromuscular Proprioceptiva, alongamento global e exercícios em turbilhão. Além disso, foi incluído um recurso eletroterapêutico, por meio do uso de um ultrassom terapêutico pulsado. Entre as diversas variáveis analisadas, o indivíduo apresentou diminuição da dor em $80 \%$, porém não foram observados resultados sobre o trofismo muscular, mensurado pela perimetria. ${ }^{15}$ Isso vai de encontro ao estudo atual, haja vista que foram realizados apenas exercícios físicos terapêuticos globais, associado à crioterapia, e o indivíduo apresentou diminuição do quadro doloroso e aumento do trofismo muscular dos braços e da coxa direita. 
Após as 26 sessões de cinesioterapia, durante quatro meses, em que o indivíduo foi submetido, observou-se a melhora da percepção dolorosa dele, com consequente progresso da qualidade de vida. O que pode ser explicado pelos benefícios ofertados pela atividade física regular para os indivíduos com $\mathrm{AR}$, como a redução da dor, a melhora das condições gerais de vida, da qualidade do sono, das condições energéticas e do desempenho funcional, especialmente a longo prazo. ${ }^{22}$

Os resultados evidenciados neste estudo concordam com outros achados na literatura. Myra et al. (2015) realizaram um estudo de caso com um indivíduo do gênero feminino com $A R$ associada ao lúpus eritematoso sistêmico, onde o submeteram a 15 sessões de cinesioterapia, duas vezes por semana. A partir de exercícios de fortalecimento muscular de membros superiores, mobilização articular de punhos e tornozelos e técnicas massoterapêuticas foi possível a obtenção de redução álgica, passando de 10 para quatro pontos na escala visual analógica e melhora de cinco domínios da qualidade de vida. ${ }^{23}$ Neste estudo, observou-se diminuição da dor em cinco pontos dolorosos e da sua intensidade em três pontos, passando de um quadro de dor moderada para um quadro de dor leve, além da melhora dos domínios limitação por aspectos emocionais, dor, saúde mental, capacidade funcional e vitalidade, relacionados à qualidade de vida, do indivíduo com AR.

Os achados já mencionados ainda concordam com os registros de Dal Molin et al. (2015), onde desenvolveu-se um estudo de caso com um homem com AR. O indivíduo realizou 16 sessões de fisioterapia, duas vezes por semana, com duração de 50 minutos cada. O plano fisioterapêutico baseou-se em terapia manual, exercícios para amplitude de movimento articular, alongamentos, cinesioterapia com movimentos funcionais e fortalecimento muscular, eletroterapia (uso de ultrassom com anti-inflamatório) e analgesia com uso de gelo. Ao fim, constatou-se que houve diminuição da dor, melhora da amplitude de movimento articular e da qualidade de vida do indivíduo. ${ }^{16} \mathrm{O}$ que concorda com o estudo atual, visto que o indivíduo, que também era homem, apresentou melhora do quadro doloroso e da qualidade de vida.

Outros autores ainda relatam os benefícios da cinesioterapia sobre o quadro doloroso e a qualidade de vida de indivíduos com AR. No estudo de Jorge, Comin e Wibelinger (2016) ${ }^{13}$ um indivíduo com AR e outras doenças reumáticas foi submetido a um plano de intervenção fisioterapêutico realizado em 25 sessões, com frequência de duas sessões semanais, onde foi possível observar diminuição da intensidade da dor na fase pós-intervenção. O que concorda, ainda, com os resultados de Jorge et al. (2017), ${ }^{14}$ onde um indivíduo com AR foi submetido a 30 sessões de fisioterapia, com frequência de duas sessões semanais. Embora não tenha sido constatada diminuição do número de pontos dolorosos, houve a diminuição da intensidade da dor em cinco pontos e melhora da qualidade de vida em quatro domínios da qualidade de vida.

Além dos benefícios supracitados com relação à diminuição da dor e à melhora da qualidade de vida, o indivíduo do presente estudo apresentou aumento do trofismo muscular dos braços e da coxa direita, mensurado pela perimetria. 
Este estudo apresenta algumas limitações. Dada a predileção da AR pelo gênero feminino, pode-se justificar a baixa ocorrência da doença em homens e a carência literária de pesquisas com indivíduos do gênero masculino portadores de AR. Outro aspecto pouco abordado pela literatura foi a avaliação do trofismo muscular em indivíduos com $\mathrm{AR}$ submetidos a intervenção fisioterapêutica por meio da cinesioterapia. Dessa forma, ancorado nas evidências apre- sentadas neste artigo, sugere-se a realização de novos estudos com a mesma temática.

\section{CONSIDERAÇõES FINAIS}

O programa cinesioterapêutico apresentado neste estudo foi benéfico para a diminuição da dor, aumento do trofismo muscular dos braços e da coxa direita e melhora da qualidade de vida em um homem com AR.

\section{REFERÊNCIAS}

1. Wibelinger LM. Fisioterapia em Reumatologia, 2. ed. Rio de Janeiro (RJ): Revinter Ltda., 2014.

2. Brenol CV, Monticiflo OA, Xavier RM, Brenol JCT. Artrite Reumatoide e Aterosclerose. Rev Assoc Med Bras 2007; 5(53): 465-70.

3. Khurana R., Berney SM. Clinical aspects of rheumatoid arthritis. Pathophysiology 2005; 12(3): 153-65.

4. Kulkamp W., Dario AB, Gevaerd MS, Domenech SC. Artrite reumatoide e exercício físico: resgate histórico e cenário atual. Rev Bras Ativ Fis Saúde 2009; 14(1): 55-64.

5. Wibelinger LM, Dal Molin V., Myra RS, DeMarco M. Perfil de uma população portadora de artrite reumatoide em um município do interior do Rio Grande do Sul. Rev EFDeportes 2015; 19(202): 1.

6. Gignac MAM, Lacaille D., Beaton DE, Backman CL, Cao X., Badley EM. Striking a Balance: Work-Health-Personal Life Conflict in Women and Men with Arthritis and its Association with Work Outcomes. J Occup Rehab 2014; 24(3): 573-84.

7. Gizińska M., Rutkowski R., Romanowski W., Lewandowski J., Straburzyńska-Lupa A. Effects of Whole-Body Cryotherapy in Comparison with Other Physical Modalities Used with Kinesitherapy in Rheumatoid Arthritis. BioMed Res Int 2015; 1(2015): 1-7.

8. Owens GM. Optimizing Rheumatoid Arthritis Therapy: Using Objective Measures of Disease Activity to Guide Treatment. Am Health Drug Benefits 2015; 8(7): 354-60.

9. Iversen MD, Brawerman M., Iversen CN. Recommendations and the state of the evidence for physical activity interventions for adults with rheumatoid arthritis: 2007 to present. Int J Clin Rheumatol 2012; 7(5): 489-503.

10. Martinez JE, Grassi DC, Marques LG. Análise da aplicabilidade de três instrumentos de avaliação de dor em distintas unidades de atendimento: ambulatório, enfermaria e urgência. Rev Bras Reumatol 2011; 51(4): 304-8.

11. Wenngren A., Stalnacke BM. Computerized assessment of pain drawing area: a pilot study. Neuropsychiatr Dis Treat. 2009; 5(1): 451-6.

12. Burille A., Cervinski T., Vidmar MF, Wibelinger LM. Qualidade de vida de portadores de espondilite anquilosante submetidos a um programa de hidrocinesioterapia. Rev EFDeportes, 2012; 17(169): 1 .

13. Jorge MSG, Comin JDP, Wibelinger LM. Intervenção fisioterapêutica em um indivíduo com artrite reumatoide, lúpus eritematoso sistêmico e Síndrome de Sjögren: relato de caso. Rev. Ciênc. Méd. Biol. 2016; 15(2): 231-237. 
14. Jorge MSG, Garbin K., Müller PL, Wibelinger LM. Atuação fisioterapêutica em um indivíduo com lúpus eritematoso sistêmico associado à artrite reumatoide e à fibromialgia. ABCS Health Sci. 2017; 42(1): 60-64.

15. Almeida PA, Passos GB, Teixeira GG, Leopoldino AAO, Teixeira VP, Souza RA. Intervenção fisioterapêutica na artrite reumatoide: relato de caso. Ter Man 2009; 7(31): 221-6.

16. Dal Molin V., Myra RS, Possebom V., Vieira G., Wibelinger LM. Intervenção fisioterapêutica em paciente portador de artrite reumatoide: um estudo de caso. Rev EFDeportes 2015; 20(209): 1.

17. Rosa CGS, Heringer DM, Silva PCC. A eficácia da hidroterapia em pacientes com artrite reumatoide: estudo de revisão. Rev Amazon Science \& Health 2015; 3(1): 37-41.

18. Nijs J., Kosek E., Van Oosterwijck J., Meeus M. Dysfunctional endogenous analgesia during exercise in patients with chronic pain: to exercise or not to exercise? Pain Physician 2012; 15(3): 205-13.

19. Østerås N., Kjeken I. A tailored hand exercise program improves function of the rheumatoid hand. J Physiother 2015; 61(2): 96.

20. Iop RR, Shiratori AP, Ferreira L., Júnior NGB, Domenech SC, Gevaerd MS. Capacidade de produção de força de preensão isométrica máxima em mulheres com artrite reumatoide: um estudo piloto. Fisioter Pesq 2015; 22(1): 11-6.

21. Adams J., Bridle C., Dosanjh S., Heine P., Lamb SE, Lord J., et al. Strengthening and stretching for rheumatoid arthritis of the hand (SARAH): design of a randomised controlled trial of a hand and upper limb exercise intervention - ISRCTN89936343. BMC Musculoskelet Disord, 2012; 13(230): 1 .

22. Middleton KR, Ward MM, Haaz S., Velummylum S., Fike A., Acevedo AT, et al. A pilot study of yoga as self-care for arthritis in minority communities. Health Qual Life Outcomes 2013; 11(55): 1.

23. Myra RS, DeMarco M., Zanin C., Wibelinger LM. Intervenção cinesioterapêutica na qualidade de vida, dor e força muscular de paciente portador de artrite reumatoide e lúpus eritematoso sistêmico. Relato de caso. Rev Dor 2015; 16(2): 153-5.

\section{DAdos dos Autores}

\section{BRUNA KNOB}

Graduanda do Curso de Fisioterapia da Universidade de Passo Fundo. Passo Fundo/RS - Brasil. brunaknob33@gmail.com

\section{Matheus Santos Gomes Jorge}

Fisioterapeuta e pós-graduando do Curso de Especialização em Fisioterapia Traumato-ortopédica da Universidade de Passo Fundo. Passo Fundo/RS - Brasil. mathjorge5@gmail.com

\section{FERnANdA LORENZet Bresolin}

Graduanda do Curso de Fisioterapia da Universidade de Passo Fundo. Passo Fundo/RS - Brasil. fernanda-bresolin@outlook.com

\section{Leticia Aparecida Bolzan}

Graduanda do Curso de Fisioterapia da Universidade de Passo Fundo. Passo Fundo/RS - Brasil. leticiabolzan57@gmail.com 


\section{DAFNe dos Santos Ribeiro}

Graduanda do Curso de Fisioterapia da Universidade de Passo Fundo. Passo Fundo/RS - Brasil. daafne.ribeiro@gmail.com

\section{Caroline Zanin}

Graduanda do Curso de Fisioterapia da Universidade de Passo Fundo. Passo Fundo/RS - Brasil. carolczanin@gmail.com

\section{Lia Mara Wibelinger}

Doutora em Gerontologia Biomédica pela Pontifícia Universidade Católica/RS. Docente do Curso de Fisioterapia e do Programa de Pós-graduação em Envelhecimento Humano da Universidade de Passo Fundo. Passo Fundo/RS - Brasil liafisio@yahoo.com.br

Submetido em: 10-4-2017

Aceito em: 19-7-2017 